\title{
Identifying off-diagonal communities using the Australian Early Development Census results
}

Robert Tanton ${ }^{1}$,Melanie Dare ${ }^{2}$, Sally Brinkman ${ }^{3,4}$, Billie-Giles Corti ${ }^{5}$, Ilan Katz ${ }^{6}$, Geoff Woolcock ${ }^{7}$, Sharon Goldfeld ${ }^{8}$

1. NATSEM, Institute for Governance and Policy Analysis, University of Canberra, Bruce ACT 2601, Australia, robert.tanton@ canberra.edu.au (corresponding author)

2. Institute for Governance and Policy Analysis, University of Canberra, Bruce ACT 2601, Australia, lain.dare@canberra.edu.au

3. Fraser Mustard Centre, Telethon Kids Institute, The University of Western Australia, Subiaco WA 6008, Australia. sally.brinkman@telethonkids.org.au

4. School of Population Health, The University of Adelaide, Adelaide SA 5005, Australia.

5. McCaughey VicHealth Community Wellbeing Unit, Melbourne School of Population and Global Health, the University of Melbourne, Melbourne, VIC, 3010, Australia, b.gilescorti@unimelb.edu.au

6. Social Policy Research Centre, University of New South Wales, ilan.katz@ unsw.edu.au

7. Wesley Mission Brisbane, Chermside QLD 4032, Australia g.woolcock@ wmb.org.au

8. Murdoch Childrens Research Institute, Royal Children's Hospital, Melbourne, sharon.goldfeld@ rch.org.au

\section{Identifying off-diagonal communities using the Australian Early Development Census results}

\section{Keywords}

Indicators, built environment, child development, socio-economic standards 


\begin{abstract}
An individual's, and indeed the nation's, social and economic futures are highly dependent on Early Childhood Development (ECD) outcomes, with poor ECD inhibiting future opportunities. Ecological ECD literature describes family, community and institutions as being key factors in children's wellbeing, with suggestions that community factors may ameliorate impacts of poor ECD. It is therefore important to develop a greater understanding of those modifiable factors that positively, and negatively, affect ECD outcomes so as ECD policy and practice can be designed and implemented effectively. One approach to this analysis is through the identification and analysis of influencing factors identified within off-diagonal communities - that is those communities where children have either developed well in consideration of their highlevels of socio-economic disadvantage, or developed poorly in consideration of their low levels of socio-economic disadvantage. In this paper we describe a new method for the identification of off-diagonal communities. The method provides clear and transparent approach to community selection, including a range of methods to further interrogate the community selection ensuring a rigorous and considered selection process. This new method, based on Census and Australian Early Development Census (AEDC) data, provides the first step in identifying community factors likely to facilitate childhood wellbeing. These findings could inform policy making to reduce inequities by assisting in policy and service delivery design targeted to community needs.
\end{abstract}

\title{
Introduction
}

Early childhood development (ECD) is critical for individual, community and national health and economic outcomes. Hertzman (2010) shows that factors including the family, the community, and institutional and service settings are key determinants of children's wellbeing and psychosocial outcomes. Other research has found that poor ECD can result in children falling behind in health and education, inhibiting future social and economic opportunities (Shonkoff and Boyce, 2009). Other research suggests that community (or neighbourhood) level factors may mediate the impacts of disadvantage on ECD (see Kohen et al., 2008; Leventhal and Brooks-Gunn, 2000). As a consequence, the consideration of multiple contexts and potentially interacting community factors influencing ECD is important in understanding childhood development and inequality (Goldfeld et al, 2014; Leventhal and Brooks-Gunn, 2000). Recent work in Australia has shown that ECD is just as important for Australian children (Goldfeld et al, 2010).

However it is often difficult to separate the developmental effects of family influences from community influences because of 'selection effects'; families typically choose where they live based on affordability and community characteristics (e.g. parents often choose communities with access to good schools and/or other services which in turn may positively influence development outcomes) (Ellen and Turner, 1997; Leventhal 
and Brooks-Gunn, 2003). This interaction of factors emphasises the need to move beyond single dimension community studies, to explore the relationships between children, families and communities and the influence of such relationships on ECD (Swisher, 2008). Community planning and policy development at all levels of government would be enhanced by a better understanding of whether there are systematic community level factors that contribute to ECD, and the interactions between these and other factors (Goldfeld et al, 2014).

In order to identify community level factors influencing ECD we first need to identify where children are developing well and where they are developing poorly. The extensive ECD literature often assumes that children living in less socio-economically disadvantaged communities will develop better, on average, than those children living in disadvantaged communities. However, this is not always the case. Although in the majority of cases the wellbeing of young children in communities is consistent with the level of community disadvantage (what we term in this paper as 'on diagonal'), 'offdiagonal' communities (i.e. disadvantaged communities where ECD is unexpectedly good, or less disadvantaged communities where ECD is unexpectedly poor) provide an opportunity to explore the extent to which community level factors influence ECD (see Hertzman, 2012).

Conducting an in depth qualitative and quantitative investigation in off-diagonal disadvantaged communities can help to identify common community level factors in disadvantaged communities that result in children having better than expected Australian Early Development Census (AEDC) outcomes; and community level factors in less disadvantaged communities that result in children having worse than expected AEDC outcomes. This can then inform policy aimed at increasing AEDC results. This is the primary purpose and objective of the Kids in Communities Study (KiCS) which commenced in 2013. Community level factors hypothesized to be important have been previously identified (see Goldfeld et al, 2010) in a pilot study for KiCS.

Off diagonal communities would best be compared with nearby on-diagonal communities with similar levels of disadvantage, to facilitate comparison with communities that share the same broad local government services.

Using Hertzman's instrumental studies in North America, this paper develops a new approach to the identification of off-diagonal communities. Hertzman's approach provides an excellent base given its acknowledgement and integration of the core factors affecting ECD, including family income, parental education, parental style, neighbourhood safety, neighbourhood cohesion, neighbourhood socioeconomic status and access to quality services (childcare, programs, policies) (Hertzman, 2012). However, Hertzman identified off-diagonal communities by identifying communities that were far from a correlation line using a scatter plot. Given the importance of positive ECD for human capital and future community and economic development, and 
the growing recognition of the influence of neighbourhood in ECD, a more effective identification of neighbourhood influences on ECD is required to guide effective planning and policy development. In this paper, we therefore seek to extend Hertzman's methodology using statistical analyses to develop a more rigorous tool to identify offdiagonal communities.

The application of such an approach for the Australian context is feasible given the availability of data on many of these factors for communities across Australia, including the AEDC data available from the federal Department of Education, and SocioEconomic Indexes for Areas (SEIFA) data available from the Australian Bureau of Statistics. When combined, these datasets enable an investigation of complex neighbourhood associations at a scale suitable for the identification of modifiable community level factors that influence children's ECD.

This paper attempts to answer the question of whether a method can be developed that can identify off-diagonal communities using the AEDC and SEIFA data. The method developed in this paper identifies those communities where there are low levels of developmental vulnerability despite high levels of socio-economic disadvantage (offdiagonal-positive), and vice versa: i.e., those communities where there is a high level of developmental vulnerability despite low levels of socio-economic disadvantage (offdiagonal-negative). The method uses a matrix of the number of children in each quintile of the AEDC/SEIFA scores. The identified communities are then assessed further to ensure that the reason they were identified is not because there was one strong socioeconomic factor in that community (for example, it was close to a University so it had many low income but highly educated students).

The paper begins with a brief overview of the data used in the methods, including the AEDC and the core variables used within early childhood vulnerability analysis. The methods used in this study are then described, followed by the identification of offdiagonal communities suited for inclusion in the Kids in Communities (KiCS) study. The implications of these methods for ECD theory and practice are then explored.

\section{Data and methods}

Communities with pronounced discrepancies in developmental outcomes from those predicted by socioeconomic factors may provide the best opportunities to study factors additional to socioeconomic advantage influencing childhood development, particularly community level influences (Hertzman, 2012). In order to conduct such studies we need a rigorous and accessible mechanism to identify those communities with pronounced deviations from expected development outcomes - the off-diagonal communities. This paper outlines a method for identifying off-diagonal communities using a matrix of available childhood development and community disadvantage data, including processes to ensure identified off-diagonal communities were adequately representative 
of other communities in Australia (e.g. results were not skewed by a pocket of high disadvantage in the area or other attributing factors).

Data

Two sets of data were used to identify the off-diagonal areas: the AEDC data for each community, and the SEIFA Index of Relative Socio-economic Disadvantage (IRSD).

\section{AEDC}

Every three years data is collected across the entire country in what represents a census of child development, using the Australian Early Development Instrument which is a holistic measure of child development based on the Canadian Early Development Instrument (Janus and Offord, 2007). Teachers complete the instrument for all students in their first year of full time schooling (generally aged 5 years of age). In 2012, information was collected on 96.5 per cent of all Australian children registered to commence school in 2012 (Centre for Community Child Health and Telethon Institute for Child Health Research, 2009, 2012). The data include five domains of child development - physical health and wellbeing, social competence, emotional maturity, language and cognitive development and communication skills and general knowledge. Full information regarding the data collection and the instrument has been previously documented (Goldfeld et al, 2014) and can also be found on the official Australian Government website www.aedc.gov.au. ${ }^{1}$

AEDC data are available at two levels of geography, called AEDC Communities and AEDC Local Communities. For the majority of the country AEDC Communities represent Local Government Areas, and AEDC Local Communities represent suburbs. In areas with low population density some AEDC Local Communities represent more than one suburb to protect privacy. A criterion for reporting AEDC data at the Local Community level includes the requirement of a minimum of 15 children and at least two teachers contribute to the data collection. In all circumstances, AEDC Local Communities represent geographical boundaries that are easily recognisable to people in the community. The AEDC dataset provided by the Department of Education also includes the Australian Bureau of Statistics SEIFA index of disadvantage which has been linked at the local community level.

Due to the settlement patterns in Australia (high density urban areas and sparsely populated remote areas), there is usually huge variation in terms of a geography's population and physical size, and the local community geography used for this project is no different. The AEDC local communities range in population from one child in their

\footnotetext{
${ }^{1}$ Note that the triennial Australian Early Development Census used to be called the Australian Early Development Index. For consistency and ease of reading, we have referred to the censuses of child development as the AEDC throughout the paper.
} 
first year of schooling to 816 , with a mean of 64 children and median of 41 . In terms of total area and population, they range from $175 \mathrm{sq} \mathrm{m}$ for some of the high density metropolitan communities in Australia, to 299,393 sq km for remote areas in the middle of Australia; and from a population of 1 in some remote communities and islands to a population of 47,721 in some inner metropolitan and regional communities. The average population is 4,216 and the median is 2,701, while the average area of the local communities is $1,510 \mathrm{sq} \mathrm{km}$ while the median is $11.77 \mathrm{sq} \mathrm{km}$, showing a very skewed distribution with many more smaller communities than larger.

The AEDC indicator used in the methodology was the percentage of children classified as developmentally vulnerable (the child has scored in the lowest 10 per cent of the national AEDC population) on one or more of the 5 developmental domain(s) (DV1). In this paper, this is called DV1\%. Vulnerable children are categorised as those falling below the benchmark on any one of the five domains.

For this analysis, the requirement of a minimum of 15 children in an area was increased to 35 children. This was done to reduce the potential variability of ratios calculated in the analysis - with a child population of 15 , an extra 1 child developmentally vulnerable will lead to an increase in the proportion developmentally vulnerable of 6.6 per cent, whereas using a cut-off of 35 reduces this to less than 3 per cent. A similar method and cut off was used by (Tanton et al, 2010) in their child social exclusion index.

To date, there have been two waves of the AEDC in Australia - Wave 1 in 2009 and Wave 2 in 2012. Both sets of data were used for identifying off-diagonal communities within this study.

\section{SEIFA}

The ABS Socio-Economic Index for Areas (SEIFA) comprises of a number of indexes of disadvantage (ABS, 2013). For this work, we have used the SEIFA Index of Relative Socio-Economic Disadvantage (IRSD) as the literature on neighbourhood effects suggests that it is disadvantage that affects child outcomes, rather than advantage acting as a protective factor (hence we did not use the SEIFA Index of Relative Advantage/Disadvantage). The IRSD is calculated using a principal components analysis of a range of indicators including low income, low education, low occupation, public housing and fluency in English (for more information see Australian Bureau of Statistics (2013). SEIFA is calculated on the basis of the Australian Bureau of Statistics population Census which is conducted once every 5 years. For the purposes of this paper we have utilised the IRSD based on the 2006 Australian Census. This index provides a similar summary of the indicators used by Hertzman in his identification of off-diagonal communities (Hertzman, 2012). 


\section{Identifying the Off-diagonal and On-diagonal Communities}

To identify the most disadvantaged areas in each State, the IRSD was broken down into quintiles calculated on the basis of the range within each State (rather than between all States). This was due to differences in the range of disadvantage between the States. The Australian Capital Territory (ACT), for example, is generally less disadvantaged than other states using the SEIFA IRSD, so if we had compared SEIFA IRSD between all States no ACT communities would have been identified as disadvantaged.

The IRSD and AEDC area level data were then allocated to quintiles. Quintiles are normally calculated with an equal number of areas in each quintile. However, this means an area with 40 children gets the same weight in the quintile calculation as an area with 200 children. In this study we used child population weighted quintile, so an area with more children has a greater effect on the quintile calculation than others. This is a similar method used by Tanton et al (2010) in the Australian Child Social Exclusion index. These child weighted quintiles provide an equal number of children in each quintile group, rather than an equal number of areas.

The child weighted SEIFA IRSD and AEDC DV1\% quintiles, calculated for each State and Territory, were used to create a matrix of developmental vulnerability against socioeconomic disadvantage, from which the off-diagonals become apparent (Table 1). This matrix was created for the AEDC two waves (2009 and 2012). In both years, the 2006 SEIFA IRSD data was used (the 2011 SEIFA IRSD data was not available for the 2012 AEDCC).

\section{[Insert Table 1 about here]}

In Table 1 ' $\mathbf{A}$ ' represents those communities with a high level of socio-economic disadvantage and a low proportion of developmental vulnerable children, that is these communities' children are developing better than expected given the disadvantage in their communities and are hence classified as Off-Diagonal Positive. 'B' represents those communities with low levels of disadvantage and a high proportion of children developmentally vulnerable on one or more domain. Here the child development is poorer than expected given the low level of disadvantage in their communities and are hence classified as $\boldsymbol{O} f \boldsymbol{f}$-Diagonal Negative. ' $\mathbf{C}$ ' represents those communities with a low level of socio-economic disadvantage and a low proportion of developmental vulnerable children; that is these communities' children are developing as expected given the lack of disadvantage in their communities and are hence classified as $\boldsymbol{O n}$ Diagonal Not Disadvantaged. 'D' represents those communities with high levels of disadvantage and a high proportion of children developmental vulnerable on one or more domain. Here the child development is as expected given the high level of 
disadvantage in their communities and are hence classified as $\boldsymbol{O n - D i a g o n a l}$

\section{Disadvantaged.}

Note that due to the use of the SEIFA index in this step, the reader knows that the off diagonal communities are either disadvantaged, or not disadvantaged.

Once off diagonal communities were identified, the next step was to use the two years of AEDC data (2009 and 2012) to identify communities that were consistently offdiagonal; and communities that had swapped from being off-diagonal to on-diagonal, or on-diagonal to off-diagonal. In terms of our overall research question in identifying why communities are off-diagonal, the off-diagonal communities in both years provided some consistency; and communities that had swapped may be more interesting to study as identifying something that had changed over this period may provide insights into what contributes to being an off-diagonal community. Both types of community were of interest for this research.

Each community was therefore categorised based on the quintile they were in for each wave. These classifications identified off diagonal communities in both years; off diagonal communities in one year; changing communities (so those that went from off to on diagonal and vice versa); on diagonal communities in both years; and on diagonal communities in one year. When selecting areas to analyse, priority was given (1) to areas that were off diagonal in both years; (2) to those that had swapped; and (3) those that were off diagonal in Wave 2 (2012).

The next step was to map the off diagonal and on diagonal communities to identify communities that were geographically close, or in clusters. If communities were in close proximity to each other it was assumed that they are likely to share services (e.g. childcare, early childhood programs) and governance arrangements (e.g. local government). Equally, differences between communities to be studied were minimised potentially enabling community factors influential for ECD to be identified. An example of this for a community in Australia (Community 1) is shown in Figure 1. It can be seen from this map that this community was an off-diagonal community in Wave 2 with high disadvantage but good AEDC. Near it are two on-diagonal communities; Community 2 (good AEDC, low disadvantage) and Community 3 (low AEDC, high disadvantage). Note that there are no off diagonal: Bad AEDC, low disadvantage communities shown in this map. Given that the analysis is primarily focused on differences in AEDC, Community 3 would look like the best comparison community for Community 1.

\section{[Insert Figure 1 about here]}

Figure 1. Australian On and Off Diagonal areas, Wave 2 
Verifying the Off-diagonal Communities

After identifying potential off-diagonal communities, a number of methods were used to ensure that:

a) the SEIFA IRSD was not affected by a pocket of extreme disadvantage in the larger area; or

b) there was not something about the community's socio-economic characteristics that meant it was different to other communities in the area, and hence inappropriate for this study (see Hertzman et al, 2012). An example might be a community close to a university with a high proportion of university students with low incomes but high education.

The first issue was checked by examining the SEIFA IRSD value for the smallest available geography in each of the AEDC geographies (local communities). The smallest available geography for the SEIFA IRSD was the statistical area 1 (SA1), which consists of approximately 200 to 800 persons (Australian Bureau of Statistics, 2011). The SA1 geographies were mapped with the AEDC local community boundaries to identify areas where pockets of high or low disadvantage may have influenced the SEIFA IRSD result for that geography. The map for one of the off-diagonal communities identified (Community 1 ) is shown in Figure 2. The map shows the SA1 level SEIFA IRSD value (shades of blue with highest level of disadvantage the lightest colours grading to low levels of disadvantage as dark colours) within the AEDC geography. It can be seen that this community contains a large number of highly disadvantaged SA1 geographies and three very small low disadvantaged SA1's, and hence is not influenced by a pocket of low disadvantage. Note that for the ABS SEIFA index, lower scores are communities with greater disadvantage, whereas higher scores are communities experiencing less disadvantage.

\section{[Insert Figure 2 about here]}

Figure 2: Map of SA1 level SEIFA for Community 1

Analysing whether a community was different to nearby communities due to one indicator was undertaken because the IRSD is a summary index, combining a number of indicators of disadvantage. This means that there may be just one indicator with a very high weight in the IRSD index that is extreme in that community and influences the IRSD. Looking at the separate indicators for a community can show more detail about why the community was disadvantaged, and can highlight whether there was one indicator influencing the IRSD result for the area. 
This analysis was undertaken by mapping the AEDC boundaries with indicators of disadvantage from the Census, following the work of Hertzman and colleagues (2012). The indicators chosen for inclusion in the analysis were based on the literature regarding early childhood development (see Goldfeld et al, 2014; Hertzman et al., 2012) and included the:

- Proportion of people working full time but earning under the minimum wage (so gross individual income of less than $\$ 600$ per week). The minimum wage in Australia in 2014 was is $\$ 640.90$ per week (Fair Work Commission, 2014), so the closest Census income classification to this was $\$ 600$ per week.

- Proportion of people who did not complete a year 12 education. The base population is those who are not at school.

- Proportion of people working full time or part time in a low status occupation (labourers). The base population is those people with an occupation, so still working.

- Proportion of sole parent families.

All of these indicators were also in the IRSD, so this method was essentially decomposing the IRSD to identify whether it was being driven by one of these indicators. This method plots the previously identified off-diagonal communities with a pie chart of these indicators sourced from Census data for each community. This was done using the QGIS open source mapping software and was only done for the offdiagonal communities identified.

Note that the pie charts do not show the proportion of people who did not complete a year 12 education (for example) in that area compared with another area; they show the relative importance of the proportion of people who did not complete a year 12 education compared with the other indicators. The variables are standardised to get $100 \%$ across all the indicators presented, and then plotted on the pie chart. A worked example is shown in Table 2 for two example communities.

It can be seen that in Community 1, while the proportion of people who did not complete a year 12 education was higher than Community 2 (58\% compared to $47 \%$ ), the relative importance of this indicator was lower in Community 1 (47\% compared to $51 \%$ ). Looking at this another way, the proportion of people who did not complete a year 12 education in Community 1 was higher simply because disadvantage was generally much higher, so all the indicators were higher. Once the indicators were standardised to 100, the relative importance of people who did not complete a year 12 education in Community 1 was much lower, and was lower than the contribution of low education in Community 2.

Note that the totals in Table 2 have no relevance except as denominators to convert the indicator values to comparable scales. People in each community can contribute to more than one indicator (so they may be both low status occupation and low income). The 
values across the whole dataset range from 0 to 300 in the most disadvantaged community.

\section{[Insert Table 2 about here]}

The map for Community 1 is shown in Figure 3 which shows on and off diagonal areas (purple with none of these areas on this map, red, green and blue); areas which are not on or off diagonal (white); and pie charts where a pink slice of the pie is the importance of no year 12; a blue slice is the importance of low income; the orange slice is the importance of sole parent families; and the green slice is the importance of low status occupation. Areas with no pie charts are those where there were less than 35 children in the area doing the AEDC, so they were excluded from the analysis. As can be seen, there is very little difference in the Census data variables for the off-diagonal positive Community 1 compared with other communities nearby. Similar to Figure 1, there are no off diagonal: Bad AEDC, low disadvantage communities shown in this map.

\section{[Insert Figure 3 about here]}

Figure 3: Map of AEDC with pie chart of disadvantage for Community 1 and surrounding areas

Figure 4 shows another example where Community 4 shows the relative impact of the proportion of people who did not complete a year 12 education is much lower than elsewhere, but the relative impact of low income is much higher. Community 4 is where several tertiary education institutions are located and hence the community is influenced by the high student population in this area, which explains the low influence of the proportion of people who did not complete a year 12 education and the high influence of the proportion of people with low income. This is a classic example of where this method has highlighted an area where the IRSD seems to be influenced by one indicator, and so this area should probably not be considered as a comparison community in this study.

\section{[Insert Figure 4 about here]}

Figure 4: Map of AEDC with pie chart of disadvantage for Community 4 and surrounding areas 
After this analytic work was completed, the final choice of communities was then made in discussion with State government representatives, industry partners and other stakeholders like not-for-profit organisations who had detailed information about the service delivery and demographic makeup of each of these communities (for example, the Smith Family and the Australian Research Alliance in Children and Youth, ARACY) and had been invited to participate in state-based advisory groups for the KiCS project. Given that most States contained a number of potential off-diagonal communities, these discussions provided important background information on the communities - for example, in the ACT, there were a number of off-diagonal communities that were already the subject of different studies, so the result of these discussions was to investigate an off-diagonal community in a different region of the ACT. In each case the criteria for selection and the rationale for the final selection of communities were carefully documented.

This methodology presents a clear and rigorous process by which off-diagonal communities can be identified. The following section details the resulting off-diagonal communities identified by the matrix method.

\section{Results}

The method described above was applied to four States and one Territory in Australia. This provided a mix of capital cities, regional and remote areas.

The number of off-diagonal and on-diagonal communities in the four States and the ACT using Wave 2 of the AEDC data is shown in Table 3 with off diagonal communities shown in dark (red), on diagonal communities shown with a light shading (green), and the main diagonal (where we would expect most areas to be) highlighted in bold. It can be seen that there are many more off diagonal high disadvantage, low AEDC vulnerable communities compared to off diagonal low disadvantage, high AEDC vulnerable communities. Not surprisingly, there are many more on-diagonal communities than off-diagonal.

\section{[Insert Table 3 about here]}

For confidentiality reasons, the list of off-diagonal communities has not been made available in this paper. A number of off-diagonal communities were identified in all Australian States in the analysis except South Australia, where only 1 community was identified. One of the advantages of the method described in this paper is that if more off-diagonal areas are needed, then the next quintile for AEDC and SEIFA IRSD can be used. For South Australia, some areas came up in the AEDC Quintile 4 (vulnerable 
AEDC) and SEIFA IRSD Quintile 5 (low disadvantage). Table 3 shows a much greater number of on-diagonal communities.

The next stage was to identify whether any off-diagonal communities were off-diagonal due to a pocket of disadvantage in the community, as shown with the map in the method section. Only one of the 24 off diagonal communities identified showed pockets of disadvantage, and this is shown as Community 5 in Figure 5. As can be seen in Figure 5 , there is a pocket of low disadvantage to the North-East, and areas of higher disadvantage to the South-West.

\section{[Insert Figure 5 about here]}

Figure 5: SEIFA SA1 value for Community 5

The next step was to look at the characteristics of each area in terms of other measures of disadvantage, as described in the method section. This analysis showed up some ondiagonal areas with high levels of education and low incomes, as shown with Community 4 in Figure 4, but no off-diagonal areas.

The next step was to use maps to ensure that each off-diagonal area had a nearby ondiagonal area as a comparison. A number of areas were excluded at this step because there were no nearby on-diagonal comparison areas, including a number of areas in regional Australia.

The final step was to take the list of communities to a number of external organisations who were practitioners in the field. This step provided local context to the choice of communities. It should be noted that the external organisations were not choosing the communities from all communities in the State - they were providing contextual information on the communities selected by the statistical process. This contextual information was essential in selecting a set of communities that were willing to work with this project. In one case, a set of communities was rejected due to information from the external organisation that the communities were already involved in two other research projects, so finding willing participants for this project would be difficult.

Overall, the method identified a number of off-diagonal and nearby on-diagonal communities for further study. If an area was not identified, the criteria for identifying off diagonal communities could be relaxed to include the next quintile of developmental vulnerability and disadvantage.

This approach was required in South Australia, where no local communities were in highest quintile of disadvantage and highest quintile of AEDC or the lowest quintile of 
disadvantage and the lowest quintile of the AEDC. Hence, for South Australia only, we selected off diagonals that had the highest quintile of disadvantage and second highest quintile of the AEDC

\section{Discussion}

Compared with the method suggested by Hertzman (2012), this method provides a statistical means of identifying off diagonal communities, rather than examining a graph and subjectively identifying communities beyond a certain line. We have derived and tested a statistical method for identifying off-diagonal communities using AEDC and an index of disadvantage. It also starts to identify the reason for the off-diagonal status in the community by examining other socio-economic data. This ensures that the explanation for the off-diagonal status of the community does not arise out of demographic peculiarities such as pockets of disadvantage that influence the disadvantage index; or one aspect of disadvantage that is high in the community, and is therefore more likely to result from community factors such as physical factors, governance or social capital.

One of the main findings from this work is that there are a number of off-diagonal communities in most States in Australia, and there is usually a nearby on-diagonal community to use as a comparison. South Australia was the only State where an offdiagonal community could not be identified, but the advantage of the method we have developed is that the quintile cut-offs used to identify an off-diagonal community can be adjusted. So rather than looking at communities in quintile 1 of AEDC and quintile 1 of SEIFA we can move to the second quintile in each criteria. This will then help identify off-diagonal communities to study in all States.

While this method has been used to identify off-diagonal communities in terms of their educational outcomes, it could also be applied to other indicators. For example, it could be used to identify less disadvantaged areas with high crime rates or disadvantaged areas with low crime rates. The main point is that the method we have developed acts as an identification method - there is no causality or explanation of strong and poor ECD provided by the method. Further research must be done in the communities to explain why they are off-diagonal.

In this Kids in Communities study, now that these communities have been identified, further research will be conducted to identify why they are off diagonal, and any protective factors in a community that has a good AEDC in a disadvantaged community. This is the next stage of this project, with the methods outlined in (Goldfeld et al, 2014). Future work will look at the characteristics of these areas, including examining the distribution of these off and on diagonal areas in urban, regional and remote areas of Australia. 


\section{Conclusions}

This paper has developed a method of identifying off-diagonal communities that could be used to examine the impact of community level effects on outcomes in a range of domains. The method provides clear cut-offs for each of the two indicators, and then provides a number of methods to filter out areas where the off diagonal can be explained by factors such as a cluster of disadvantaged people or a high level for a particular indicator due to an external factor, for example, high education due to proximity to a university. This makes it possible to identify those factors within communities which are likely to facilitate better wellbeing of children and which could be modified in similar communities that do not have high levels of child wellbeing.

One of the advantages of the method is that it is possible to relax the quintile restriction slightly to identify enough off-diagonal communities required for comparison with similar communities that are on diagonal.

Once these communities have been identified, further study can be used to identify what neighbourhood factors contribute to the high AEDC in the disadvantaged off-diagonal communities and the low AEDC in the off-diagonal not disadvantaged communities. These findings could inform policy making to reduce inequities by assisting disadvantaged on-diagonal communities i.e., those that are disadvantaged with a low AEDC.

\section{References}

Australian Bureau of Statistics. 2011.

Australian Bureau of Statistics. 2013. Socio-Economic Indexes for Areas (SEIFA) 2011: Technical Paper. Cat No. 2033.0.55.001, ABS: Canberra.

Centre for Community Child Health, \& Telethon Institute for Child Health Research. 2009. A snapshot of early childhood development in Australia: AEDI National report 2009, Australian Government: Canberra.

Centre for Community Child Health, \& Telethon Institute for Child Health Research. 2012. A snapshot of early childhood development in Australia: AEDI National report 2012, Australian Government: Canberra.

Ellen I, Turner M. 1997. Does neighbourhood matter? Assessing recent evidence. Housing Policy Debate 8 : 833-866. DOI 10.1080/10511482.1997.9521280.

Fair Work Commission. 2014. Draft National Minimum Wage Order 2014, Fair Work Commission: Melbourne.

Goldfeld S, Mathews T, Brinkman S, Woolcock G, Myers J, Kershaw P, Katz I, Tanton R, Wiseman J (2010). The Kids in Communities Study: Measuring community level factors influencing children's development. Report for VicHealth. Melbourne: Murdoch Children's Research Institute. 
Goldfeld, Woolcock, Katz, Tanton, Brinkman, O’Connor, Mathews, and Giles-Corti (2014), "Neighbourhood Effects Influencing Early Childhood Development: Conceptual Model and Trial Measurement Methodologies from the Kids in Communities Study," Social Indicators Research, DOI: 10.1007/s11205-014-0578-x

Hertzman C. 2012. Bringing a population health perspective to early biodevelopment: An emerging approach. In Nature and nurture in early child development, Keating DP (ed); Cambridge University Press: 217-244. DOI 10.1017/CBO9780511975394.

Hertzman C. 2010. Framework for the social determinants of early child development. In Encyclopedia on early childhood development, Tremblay RE, Barr RG, Peters RD, Boivin M. (eds.); Centre of Excellence for Early Childhood Development: Montreal. http://www.child-encyclopedia.com/

Janus M, Offord D. 2007. Development and psychometric properties of the early development instrument (EDI): A measure of children's school readiness. Canadian Journal of Behavioural Science 39 : 1-22. doi:10.1037/cjbs2007001.

Kohen DE, Leventhal T, Dahinten VS, McIntosh CN. 2008. Neighborhood disadvantage: Pathways of effects for young children. Child Development 79 : 156-169. doi: 10.1111/j.1467-8624.2007.01117.x.

Leventhal T, Brooks-Gunn J. 2000. The neighborhoods they live in: The effects of neighbourhood residence on child and adolescent outcomes. Psychological Bulletin 126 : 309-337. DOI: 10.1037//0033-2909.126.2.309.

Leventhal T. Brooks-Gunn J. 2003. Children and youth in neighbourhood contexts. Current Directions in Psychological Science 12 : 27-31. doi: 10.1111/1467-8721.01216

Shonkoff JP, Boyce WT. 2009. Neuroscience, molecular biology, and the childhood roots of health disparities: Building a new framework for health promotion and disease prevention. Journal of the American Medical Association 301 : 2252-2259. DOI 10.1001/jama.2009.754.

Swisher R. 2008. Neighborhoods and youth: How neighborhood demographics and social processes affect youth outcomes. The Prevention Researcher 15 : 7-11.

Tanton, R, Harding, A, Daly, A, McNamara, J and Yap, M (2010). Australian Children at Risk of Social Exclusion: A Spatial Index for Gauging Relative Disadvantage. Population, Space and Place 16 (2): 135-150. 
Table 1. Off-diagonal matrix

\begin{tabular}{|c|c|c|c|c|c|c|c|}
\hline & & & \multicolumn{5}{|c|}{ SEIFA IRSD Quintile } \\
\hline & & & \multicolumn{2}{|c|}{$\begin{array}{l}\text { High level of } \\
\text { disadvantage }\end{array}$} & & \multicolumn{2}{|c|}{$\begin{array}{l}\text { Low level of } \\
\text { disadvantage }\end{array}$} \\
\hline & & & 1 & 2 & 3 & 4 & 5 \\
\hline \multirow{5}{*}{ 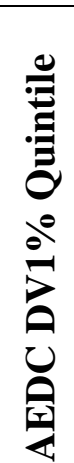 } & \multirow{3}{*}{\begin{tabular}{|l|} 
Low \% \\
Vulnerable 1 \\
or more \\
domain
\end{tabular}} & 1 & $\mathbf{A}$ & & & & C \\
\hline & & 2 & & & & & \\
\hline & & 3 & & & & & \\
\hline & \multirow{2}{*}{$\begin{array}{l}\text { High \% } \\
\text { Vulnerable } 1 \\
\text { or more } \\
\text { domain }\end{array}$} & 4 & & & & & \\
\hline & & 5 & $\mathbf{D}$ & & & & B \\
\hline
\end{tabular}


Table 2. Worked example of pie charts in maps

\begin{tabular}{lccccc}
\hline & $\begin{array}{c}\text { \% No Year } \\
\mathbf{1 2}\end{array}$ & $\begin{array}{c}\text { \% Low } \\
\text { Income }\end{array}$ & $\begin{array}{c}\text { \% Single } \\
\text { Parent } \\
\text { Family }\end{array}$ & $\begin{array}{c}\text { \% Low } \\
\text { Status } \\
\text { Occupation }\end{array}$ & Total Sum \\
\hline Raw Data & & & & & \\
Community 1 & 58 & 43 & 15 & 8 & 124 \\
Community 2 & 47 & 29 & 8 & 7 & 91 \\
\hline Data for Pie chart & & & & & \\
Community 1 & 47 & 35 & 12 & 6 & 100 \\
Community 2 & 51 & 32 & 9 & 8 & 100 \\
\hline
\end{tabular}


Table 3: Off and On Diagonal areas in four Australian States and the ACT, Wave 2

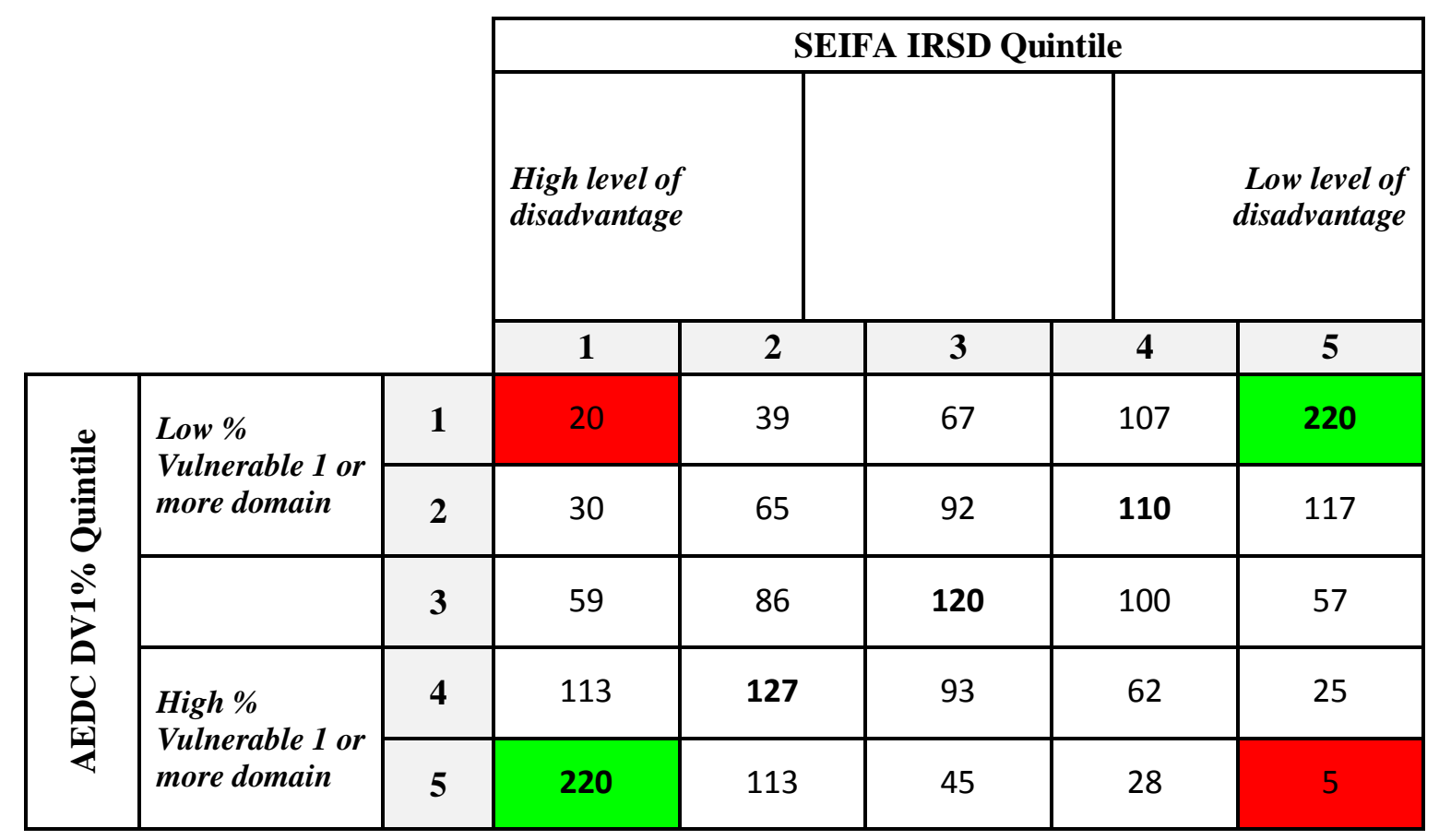

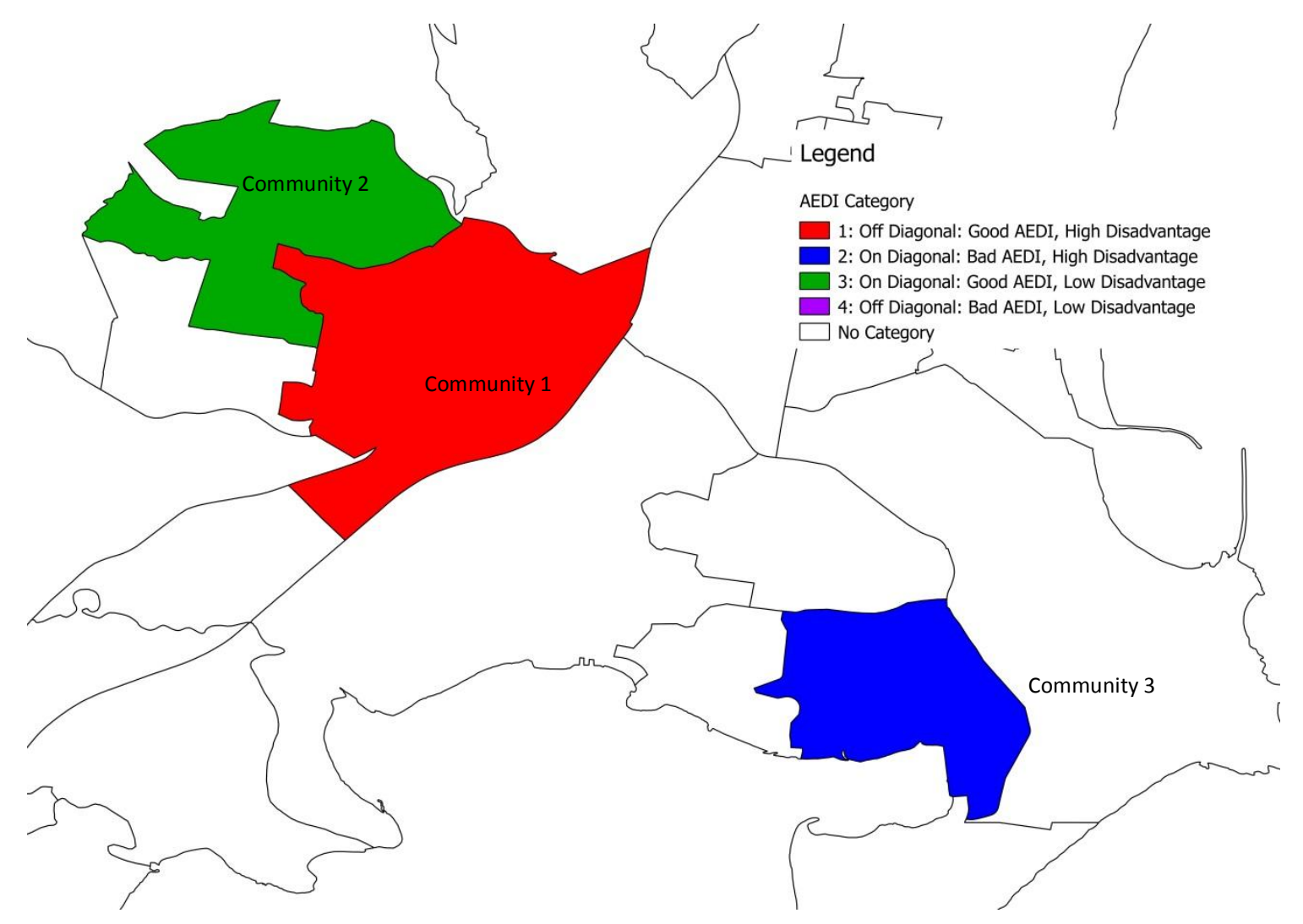



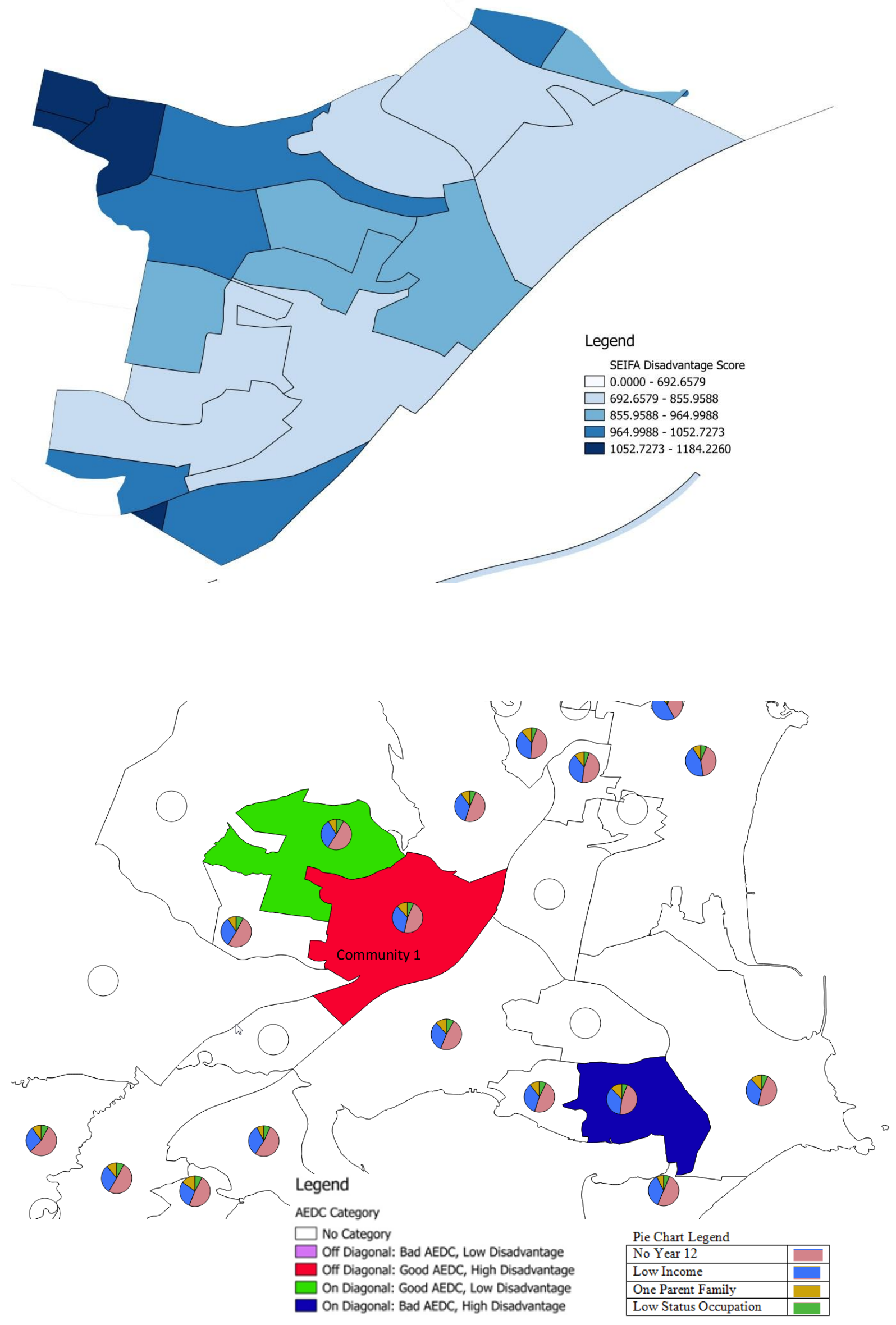

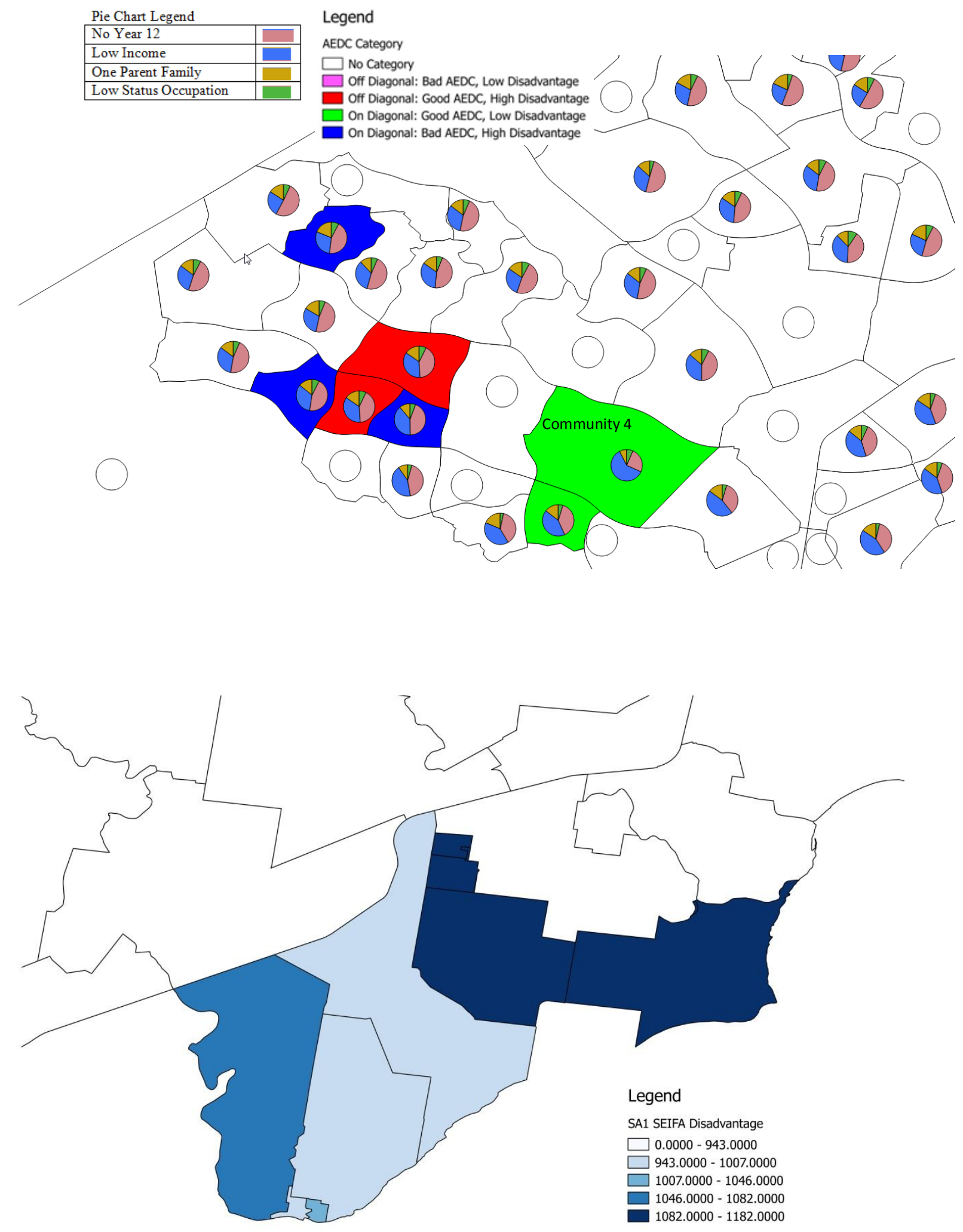
